\title{
Noncircular-PARAFAC for 2D-DOA estimation of noncircular signals in arbitrarily spaced acoustic vector-sensor array subjected to unknown locations
}

\author{
Xiaofei Zhang ${ }^{1,2,3^{*}}$, Renzheng Cao ${ }^{1}$ and Ming Zhou ${ }^{1}$
}

\begin{abstract}
s
In this paper, we propose a noncircular-parallel factor (NC-PARAFAC) algorithm for two-dimensional direction of arrival (DOA) estimation of noncircular signals for acoustic vector-sensor array. The proposed algorithm enhances the angle estimation performance via utilizing the noncircularity of the signals, and it can be suitable for arbitrary array subjected to unknown locations and achieve automatically paired two-dimensional angle estimation. The proposed algorithm has better angle estimation performance than estimation of signal parameters via rotational invariance technique, PARAFAC algorithm, and propagator method. Furthermore, the proposed algorithm has a lower computational complexity than the PARAFAC algorithm. We also derive the Crámer-Rao bound of DOA estimation of noncircular signal in acoustic vector-sensor array. The simulation results verify the effectiveness of the algorithm.
\end{abstract}

Keywords: Two-dimensional DOA estimation, Acoustic vector-sensor array, Noncircular signals

\section{Introduction}

Compared with traditional acoustic pressure sensor arrays, the acoustic vector sensors can measure the acoustic pressure as well as all three orthogonal components of the acoustic particle velocity at a single point in space, which brings about certain significant advantages in collecting more information on acoustics, better exploitation of beam forming, and enhancing the system performance [1-15]. Since the measurement model of acoustic vectorsensor array had been developed in [2], researchers mainly turned to the study on direction of arrival (DOA) estimation of incoming signals and proposed many DOA estimation algorithms, which contain Capon technique [4], propagator method (PM) $[5,13]$, estimation of signal parameters via rotational invariance technique (ESPRIT) algorithms [7-9], root-multiple signal classification (MUSIC) algorithm [10], self-initiating multiple signal classification MUSIC algorithm [11], hypercomplex MUSIC algorithm

\footnotetext{
* Correspondence: fei zxf@163.com

${ }^{1}$ College of Electronic and Information Engineering, Nanjing University of Aeronautics and Astronautics, Nanjing 210016, China

${ }^{2}$ Nanjing Panda Electronics Group, Nanjing 210002, China

Full list of author information is available at the end of the article
}

[12], parallel factor (PARAFAC) algorithm [15], successive MUSIC [14], as well as others [16-21].

The binary phase shift keying (BPSK) and amplitude modulation modulated signals, which are called noncircular signal because their statistics are rotationally variants, are widely used in communication systems $[22,23]$. As far as we know, few works on DOA estimation of noncircular signal in acoustic vector-sensor array has been reported. In this paper, we propose a noncircularPARAFAC (NC-PARAFAC) algorithm for two-dimensional (2D) DOA estimation of noncircular signals using arbitrarily spaced acoustic vector-sensor array subjected to unknown locations. Compared with PARAFAC algorithm, the proposed algorithm enhances the parameter estimation performance via utilizing the noncircularity of the signals. The proposed algorithm can be suitable for arbitrary array subjected to unknown locations and achieve automatically paired two-dimensional angle estimation. Furthermore, our algorithm has better angle estimation performance than PM, ESPRIT algorithm, and PARAFAC algorithm.

The work in [15] links the acoustic vector-sensor array parameter estimation problem to trilinear model and 
derives a PARAFAC algorithm whose angle estimation performance is better than the ESPRIT algorithm. There are some differences between our algorithm and the PARAFAC algorithm. Firstly, our algorithm exploits the noncircular property to improve DOA estimation. Secondly, our NC-PARAFAC algorithm is suitable for the DOA estimation of noncircular signal, and our algorithm can be regarded as an extension of PARAFAC. Thirdly, the real trilinear model is used for NC-PARAFAC algorithm, while the complex trilinear model is employed in PARAFAC algorithm. Our NC-PARAFAC algorithm is better for the practical system. A contribution of this paper is to extend PARAFAC method to noncircular signal acoustic vector-sensor array.

The remainder of this paper is structured as follows. Section 2 develops the data model, and Section 3 presents the algorithm and the complexity analysis. In Section 4, we derive the Crámer-Rao bound (CRB) of the DOA estimation of noncircular signal in acoustic vectorsensor array. In Section 5, simulation results are presented to verify improvement for the proposed algorithm, while conclusions are shown in Section 6.

The following notations are used: $(.)^{T},(.)^{H},(.)^{-1}$, and $(.)^{+}$ denote transpose, conjugate-transpose, inverse, and pseudoinverse operations, respectively. $\operatorname{diag}(\mathbf{v})$ stands for diagonal matrix whose diagonal element is a vector v. $D_{n}($.$) is to$ take the $n$th row of the matrix to construct a diagonal matrix. $\mathbf{I}_{K}$ is a $K \times K$ identity matrix. $\otimes$, 。, and $\odot$ are the Kronecker product, Khatri-Rao product, and Hadamard product, respectively. $\operatorname{Re}\{$.$\} and \operatorname{Im}\{$.$\} are to get the real$ part and imaginary part of the complex, respectively. $E[$. is expectation operator.

\section{Data model}

We assume that a total of $K$ narrowband plane waves impinge on an array equipped with $M$ acoustic vector sensors, which are all located at arbitrarily unknown three-dimensional positions as shown in Figure 1. We consider the noncircular signals in the far field, in which case the sources are far away enough that the arriving waves are essentially planes over the array. Also assume that the noise is an additive independent identically distributed Gaussian with zero mean and variance $\sigma^{2}$, which is independent of the sources. The $k$ th signal is arriving from direction $\left(\varnothing_{k}, \varphi_{k}\right)$, where $\varnothing_{k}$ and $\varphi_{k}$ stand for the azimuth angle and the elevation angle, respectively. Let $\boldsymbol{\theta}_{k}=$ $\left[\phi_{k}, \varphi_{k}\right]^{T}$, which is the 2D-DOA of the $k$ th source.

According to [7], the output of the irregular array containing $M$ acoustic vector sensors is

$$
\left\{\begin{array}{c}
\mathbf{p}(t)=\mathbf{A s}(t)+\mathbf{n}_{p}(t) \\
\mathbf{x}(t)=\mathbf{A} \boldsymbol{\Phi}_{x} \mathbf{s}(t)+\mathbf{n}_{x}(t) \\
\mathbf{y}(t)=\mathbf{A} \Phi_{y} \mathbf{s}(t)+\mathbf{n}_{y}(t) \\
\mathbf{z}(t)=\mathbf{A} \boldsymbol{\Phi}_{z} \mathbf{s}(t)+\mathbf{n}_{z}(t)
\end{array}\right.
$$

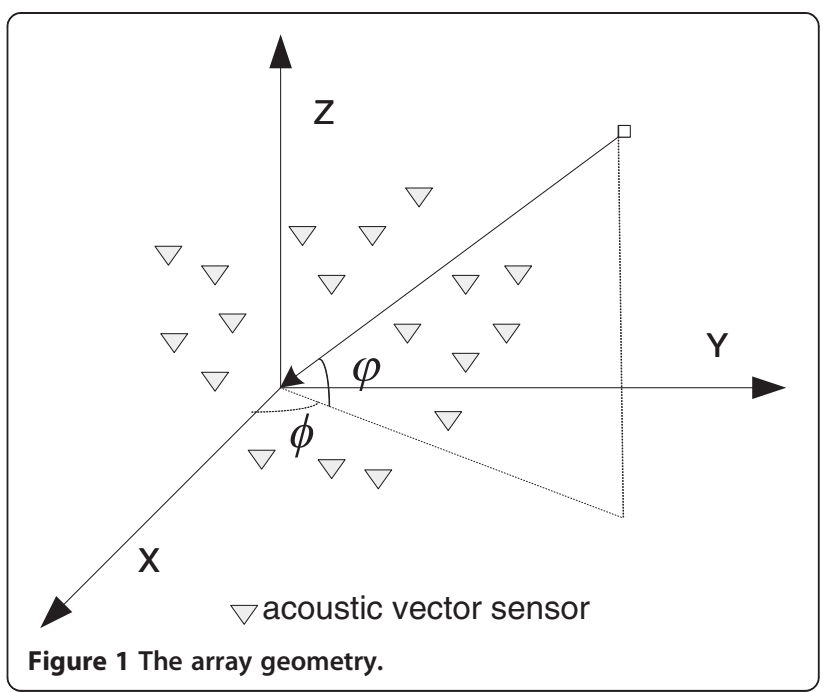

where $\mathbf{A}=\left[\mathbf{a}_{1}, \mathbf{a}_{2}, \ldots, \mathbf{a}_{K}\right], \mathbf{a}_{k}=\left[q_{1}\left(\boldsymbol{\theta}_{k}\right), q_{2}\left(\boldsymbol{\theta}_{k}\right), \ldots q_{M}\left(\boldsymbol{\theta}_{k}\right)\right]^{T}$ with $q_{m}\left(\boldsymbol{\theta}_{k}\right)=e^{j 2 \pi \tau_{m k}} \cdot \tau_{m k}$ is the differential time delay of the $k$ th wave between the origin and the $m$ th sensor; $\mathbf{s}(t)$ contains $K$ signals; $\mathbf{n}_{p}(t), \mathbf{n}_{x}(t), \mathbf{n}_{y}(t)$, and $\mathbf{n}_{z}(t)$ are the received signals from the acoustic vector-sensor array. $\boldsymbol{\Phi}_{x}$, $\boldsymbol{\Phi}_{y}$, and $\boldsymbol{\Phi}_{z}$ are shown as

$$
\begin{aligned}
& \Phi_{x}=\operatorname{diag}\left(\cos \phi_{1} \cos \varphi_{1}, \quad \cos \phi_{2} \cos \varphi_{2}, \cdots, \quad \cos \phi_{K} \cos \varphi_{K}\right) . \\
& \Phi_{y}=\operatorname{diag}\left(\sin \phi_{1} \cos \varphi_{1}, \quad \sin \phi_{2} \cos \varphi_{2}, \cdots, \quad \sin \phi_{K} \cos \varphi_{K}\right) . \\
& \boldsymbol{\Phi}_{z}=\operatorname{diag}\left(\sin \varphi_{1}, \quad \sin \varphi_{2}, \cdots, \quad \sin \varphi_{K}\right) .
\end{aligned}
$$

Consider that channel state information is constant during $L$ transmitting symbols; we have

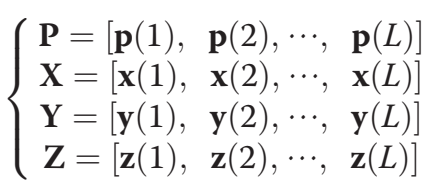

We construct the following matrix $\mathbf{X}_{E} \in \mathfrak{£}^{4 M \times L}$

$$
\mathbf{X}_{E}=\left[\begin{array}{c}
\mathbf{P} \\
\mathbf{X} \\
\mathbf{Y} \\
\mathbf{Z}
\end{array}\right]=\left[\begin{array}{c}
\mathbf{A} \\
\mathbf{A} \Phi_{x} \\
\mathbf{A} \Phi_{y} \\
\mathbf{A} \Phi_{z}
\end{array}\right] \mathbf{S}+\left[\begin{array}{c}
\mathbf{N}_{p} \\
\mathbf{N}_{x} \\
\mathbf{N}_{y} \\
\mathbf{N}_{z}
\end{array}\right]
$$

where $\mathbf{S}=[\mathbf{s}(1), \mathbf{s}(2), \cdots, \mathbf{s}(L)] \in \mathfrak{E}^{K \times L}$ is the source matrix for $L$ samples; and $\mathbf{N}_{p}=\left[\mathbf{n}_{p}(1), \mathbf{n}_{p}(2), \mathrm{L}, \mathbf{n}_{p}(L)\right], \mathbf{N}_{x}=\left[\mathbf{n}_{x}\right.$ (1), $\left.\mathbf{n}_{x}(2), \mathrm{L}, \mathbf{n}_{x}(L)\right], \mathbf{N}_{y}=\left[\mathbf{n}_{y}(1), \mathbf{n}_{y}(2), \mathrm{L}, \mathbf{n}_{y}(L)\right]$, and $\mathbf{N}_{z}=$ $\left[\mathbf{n}_{z}(1), \mathbf{n}_{z}(2), L, \mathbf{n}_{z}(L)\right]$ are the noise matrices. According to noncircular property, the source matrix can be denoted by

$$
\mathbf{S}=\psi \mathbf{S}_{0} .
$$


where $\boldsymbol{\psi}=\operatorname{diag}\left\{e^{i \psi_{1}}, \ldots, e^{i \psi_{K}}\right\}$ with $e^{i \psi_{p}} \neq e^{i \psi_{q}}$ for $p \neq q$. $\mathbf{S}_{0} \in i^{K \times L}$ with $\mathbf{S}_{0}=\mathbf{S}_{0} *$.

\section{NC-PARAFAC algorithm for 2D-DOA estimation}

\subsection{Data reconstruction}

Expanding $\mathbf{X}_{E}$, the data matrix is reconstructed and denoted as $\mathbf{Y} \in i^{8 M \times L}$

$$
\mathbf{Y}=\left[\begin{array}{c}
\operatorname{Re}\left\{\mathbf{X}_{E}\right\} \\
\operatorname{Im}\left\{\mathbf{X}_{E}\right\}
\end{array}\right]
$$

Via implementing some row transformations, $\mathbf{V}$ can be obtained and expressed by

$$
\mathbf{V}=\left[\begin{array}{c}
\operatorname{Re}\left\{\mathbf{A} \mathbf{S}+\mathbf{N}_{1}\right\} \\
\operatorname{Im}\left\{\mathbf{A} \mathbf{S}+\mathbf{N}_{1}\right\} \\
\operatorname{Re}\left\{\mathbf{A} \Phi_{x} \mathbf{S}+\mathbf{N}_{2}\right\} \\
\operatorname{Im}\left\{\mathbf{A} \Phi_{x} \mathbf{S}+\mathbf{N}_{2}\right\} \\
\operatorname{Re}\left\{\mathbf{A} \Phi_{y} \mathbf{S}+\mathbf{N}_{3}\right\} \\
\operatorname{Im}\left\{\mathbf{A} \Phi_{y} \mathbf{S}+\mathbf{N}_{3}\right\} \\
\operatorname{Re}\left\{\mathbf{A} \Phi_{z} \mathbf{S}+\mathbf{N}_{4}\right\} \\
\operatorname{Im}\left\{\mathbf{A} \Phi_{z} \mathbf{S}+\mathbf{N}_{4}\right\}
\end{array}\right]=\left[\begin{array}{c}
\operatorname{Re}\{\mathbf{A S}\}+\operatorname{Re}\left\{\mathbf{N}_{p}\right\} \\
\operatorname{Im}\{\mathbf{A} \mathbf{S}\}+\operatorname{Im}\left\{\mathbf{N}_{p}\right\} \\
\operatorname{Re}\left\{\mathbf{A} \boldsymbol{\Phi}_{x} \mathbf{S}\right\}+\operatorname{Re}\left\{\mathbf{N}_{x}\right\} \\
\operatorname{Im}\left\{\mathbf{A} \boldsymbol{\Phi}_{x} \mathbf{S}\right\}+\operatorname{Im}\left\{\mathbf{N}_{x}\right\} \\
\operatorname{Re}\left\{\mathbf{A} \boldsymbol{\Phi}_{y} \mathbf{S}\right\}+\operatorname{Re}\left\{\mathbf{N}_{y}\right\} \\
\operatorname{Im}\left\{\mathbf{A} \boldsymbol{\Phi}_{y} \mathbf{S}\right\}+\operatorname{Im}\left\{\mathbf{N}_{y}\right\} \\
\operatorname{Re}\left\{\mathbf{A} \boldsymbol{\Phi}_{z} \mathbf{S}\right\}+\operatorname{Re}\left\{\mathbf{N}_{z}\right\} \\
\operatorname{Im}\left\{\mathbf{A} \boldsymbol{\Phi}_{z} \mathbf{S}\right\}+\operatorname{Im}\left\{\mathbf{N}_{z}\right\}
\end{array}\right]
$$

The matrix $\mathbf{V} \in_{i}^{8 M \times L}$ can be also expressed by

$$
\begin{aligned}
\mathbf{V}=\left[\begin{array}{c}
{\left[\begin{array}{c}
\operatorname{Re}\{\mathbf{A} \boldsymbol{\psi}\} \\
\operatorname{Im}\{\mathbf{A} \boldsymbol{\psi}\}
\end{array}\right] \mathbf{S}_{0}+\left[\begin{array}{l}
\operatorname{Re}\left\{\mathbf{N}_{p}\right\} \\
\operatorname{Im}\left\{\mathbf{N}_{p}\right\}
\end{array}\right]} \\
{\left[\begin{array}{l}
\operatorname{Re}\{\mathbf{A} \boldsymbol{\psi}\} \\
\operatorname{Im}\{\mathbf{A} \boldsymbol{\psi}\}
\end{array}\right] \boldsymbol{\Phi}_{x} \mathbf{S}_{0}+\left[\begin{array}{l}
\operatorname{Re}\left\{\mathbf{N}_{x}\right\} \\
\operatorname{Im}\left\{\mathbf{N}_{x}\right\}
\end{array}\right]} \\
{\left[\begin{array}{l}
\operatorname{Re}\{\mathbf{A} \boldsymbol{\psi}\} \\
\operatorname{Im}\{\mathbf{A} \boldsymbol{\psi}\}
\end{array}\right] \boldsymbol{\Phi}_{y} \mathbf{S}_{0}+\left[\begin{array}{l}
\operatorname{Re}\left\{\mathbf{N}_{y}\right\} \\
\operatorname{Im}\left\{\mathbf{N}_{y}\right\}
\end{array}\right]} \\
{\left[\begin{array}{l}
\operatorname{Re}\{\mathbf{A} \boldsymbol{\psi}\} \\
\operatorname{Im}\{\mathbf{A} \boldsymbol{\psi}\}
\end{array}\right] \boldsymbol{\Phi}_{z} \mathbf{S}_{0}+\left[\begin{array}{l}
\operatorname{Re}\left\{\mathbf{N}_{z}\right\} \\
\operatorname{Im}\left\{\mathbf{N}_{z}\right\}
\end{array}\right]}
\end{array}\right]=\left[\begin{array}{l}
\mathbf{V}_{2} \\
\mathbf{V}_{3} \\
\mathbf{V}_{4}
\end{array}\right] \\
=\left[\begin{array}{c}
\overline{\mathbf{A}} \mathbf{S}_{0} \\
\overline{\mathbf{A}} \boldsymbol{\Phi}_{x} \mathbf{S}_{0} \\
\overline{\mathbf{A}} \boldsymbol{\Phi}_{y} \mathbf{S}_{0} \\
\overline{\mathbf{A}} \boldsymbol{\Phi}_{z} \mathbf{S}_{0}
\end{array}\right]+\tilde{\mathbf{N}}
\end{aligned}
$$

where

$$
\overline{\mathbf{A}}=\left[\begin{array}{c}
\operatorname{Re}\{\mathbf{A} \boldsymbol{\psi}\} \\
\operatorname{Im}\{\mathbf{A} \boldsymbol{\psi}\}
\end{array}\right] \in \mathrm{i}^{2 M \times K}
$$

We define the matrix $\mathbf{H}$ as

$$
\mathbf{H}=\left[\begin{array}{cccc}
1 & 1 & \cdots & 1 \\
\cos \phi_{1} \cos \varphi_{1} & \cos \phi_{2} \cos \varphi_{2} & \cdots & \cos \phi_{K} \cos \varphi_{K} \\
\sin \phi_{1} \cos \varphi_{1} & \sin \phi_{2} \cos \varphi_{2} & \ddots & \sin \phi_{K} \cos \varphi_{K} \\
\sin \varphi_{1} & \sin \varphi_{2} & \cdots & \sin \varphi_{K}
\end{array}\right] \in \mathrm{i}^{4 \times K}
$$

The matrix $\mathbf{V}_{n}(n=1,2, \ldots, 4)$ in Equation 10 can be denoted by

$$
\mathbf{V}_{n}=\overline{\mathbf{A}} D_{n}(\mathbf{H}) \mathbf{S}_{0}+\mathbf{N}_{n}, \quad n=1,2, \ldots, 4
$$

where $D_{n}($.$) is to extract the n$th row of its matrix argument and construct a diagonal matrix out of it. $D_{n}(\mathbf{H})=$ $\operatorname{diag}\left(\left[h_{n, 1}, h_{n, 2}, \cdots, h_{n, K}\right]\right) \in £^{K \times K}$, and $h_{m, k}$ stands for the $(m, k)$ element of the matrix $\mathbf{H}$. The noiseless signal in Equation 13 can be denoted as the trilinear model [24],

$$
\begin{aligned}
x_{m, l, n} & =\sum_{k=1}^{K} a_{m, k} s_{l, k} h_{n, k}, \quad m=1, \ldots, 2 M, l \\
& =1, \ldots, \quad L, \quad n=1,2, \quad 3,4
\end{aligned}
$$

where $s_{l, k}$ is the $(l, k)$ element of matrix $\mathbf{S}_{0}$ and similarly for the others. The trilinear model displays the reflection for three different kinds of diversity [24]. Equation 13 can be interpreted as slicing the $3 \mathrm{D}$ data in a series of slices along the spatial direction. In similar ways, the model symmetry in Equation 14 allows two more matrix system rearrangements, where we have

$$
\begin{aligned}
& \mathbf{U}_{l}=\mathbf{H} D_{p}\left(\mathbf{S}_{0}^{T}\right) \overline{\mathbf{A}}^{T}+\mathbf{N}_{l}^{\prime}, \quad l=1, \ldots, L . \\
& \mathbf{W}_{m}=\mathbf{S}_{0}{ }^{T} D_{m}(\overline{\mathbf{A}}) \mathbf{H}^{T}+\mathbf{N}_{m}^{\prime}, \quad m=1, \ldots, 2 M .
\end{aligned}
$$

where $\mathbf{N}_{l}^{\prime}$ and $\mathbf{N}_{m}^{\prime}$ are the received noises. $\mathbf{V}_{n}, \mathbf{U}_{l}$, and $\mathbf{W}_{l}$ are slices along the different directions when slicing the trilinear model.

Remark 1. The trilinear model with $M \times L \times 4$ is used in PARAFAC algorithm [19], while that with $2 M \times L \times 4$ is employed in our algorithm. Because of noncircularity, the array aperture in our algorithm can be regarded as two times that of the PARAFAC algorithm.

\subsection{Trilinear decomposition}

Trilinear alternating least square (TALS) algorithm is a common data detection method for trilinear model [24]. The detailed steps are shown as follows: Referring to Equation 10, least squares (LS) fitting can be given by

$$
\min _{\overline{\mathbf{A}}, H, \mathbf{S}_{0}}\left\|\left[\begin{array}{c}
\mathbf{V}_{1} \\
\mathbf{V}_{2} \\
\mathbf{V}_{3} \\
\mathbf{V}_{4}
\end{array}\right]-\left[\begin{array}{c}
\overline{\mathbf{A}} D_{1}(\mathbf{H}) \\
\overline{\mathbf{A}} D_{2}(\mathbf{H}) \\
\overline{\mathbf{A}} D_{3}(\mathbf{H}) \\
\left.\overline{\mathbf{A}} D_{4}(\mathbf{H})\right)
\end{array}\right] \mathbf{S}_{0}\right\|_{F}
$$

LS update for $\mathbf{S}_{0}$ is shown as

$$
\hat{\mathbf{S}}_{0}=\left[\begin{array}{c}
\overline{\mathbf{A}} D_{1}(\mathbf{H}) \\
\overline{\mathbf{A}} D_{2}(\mathbf{H}) \\
\overline{\mathbf{A}} D_{3}(\mathbf{H}) \\
\left.\overline{\mathbf{A}} D_{4}(\mathbf{H})\right)
\end{array}\right]^{+}\left[\begin{array}{l}
\mathbf{V}_{1} \\
\mathbf{V}_{2} \\
\mathbf{V}_{3} \\
\mathbf{V}_{4}
\end{array}\right]
$$

Similarly, from the second way of slices: $\mathbf{U}_{l}=\mathbf{H} D_{l}$ $\left(\mathbf{S}_{0}{ }^{T}\right) \overline{\mathbf{A}}^{T}+\mathbf{N}_{l}^{\prime}, \quad l=1, \ldots, L$, LS fitting is

$$
\min _{\overline{\mathbf{A}}, H, \mathbf{S}_{0}}\left\|\left[\begin{array}{c}
\mathbf{U}_{1} \\
\mathbf{U}_{2} \\
\vdots \\
\mathbf{U}_{L}
\end{array}\right]-\left[\begin{array}{c}
\mathbf{H} D_{1}\left(\mathbf{S}_{0}^{T}\right) \\
\mathbf{H} D_{2}\left(\mathbf{S}_{0}^{T}\right) \\
\vdots \\
\mathbf{H} D_{L}\left(\mathbf{S}_{0}^{T}\right)
\end{array}\right] \overline{\mathbf{A}}^{T}\right\|_{F}
$$


LS update for $\overline{\mathbf{A}}$ is

$$
\hat{\mathbf{A}}^{T}=\left[\begin{array}{c}
\mathbf{H} D_{1}\left(\mathbf{S}_{0}^{T}\right) \\
\mathbf{H} D_{2}\left(\mathbf{S}_{0}^{T}\right) \\
\vdots \\
\mathbf{H} D_{L}\left(\mathbf{S}_{0}^{T}\right)
\end{array}\right]^{+}\left[\begin{array}{c}
\mathbf{U}_{1} \\
\mathbf{U}_{2} \\
\vdots \\
\mathbf{U}_{L}
\end{array}\right]
$$

Finally, from the third way of slices: $\mathbf{W}_{m}=\mathbf{S}_{0}{ }^{T} D_{m}(\overline{\mathbf{A}})$ $\mathbf{H}^{T}+\mathbf{N}_{m}^{\prime}, \quad m=1, \ldots, 2 M$, LS update for $\mathbf{H}$ is shown as

$$
\hat{\mathbf{H}}^{T}=\left[\begin{array}{c}
\mathbf{S}_{0}^{T} D_{1}(\overline{\mathbf{A}}) \\
\mathbf{S}_{0}^{T} D_{2}(\overline{\mathbf{A}}) \\
\vdots \\
\mathbf{S}_{0}^{T} D_{2 M}(\overline{\mathbf{A}})
\end{array}\right]^{+}\left[\begin{array}{c}
\mathbf{W}_{1} \\
\mathbf{W}_{2} \\
\vdots \\
\mathbf{W}_{2 M}
\end{array}\right]
$$

The sum of squared residuals (SSR) in the trilinear fitting is defined as

$$
\mathrm{SSR}=\sum_{m=1}^{2 M} \sum_{l=1}^{L} \sum_{n=1}^{4}\left|\tilde{x}_{m, l, n}-\sum_{k=1}^{K} \hat{a}_{m, k} \hat{s}_{l, k} \hat{h}_{n, k}\right|^{2} .
$$

where $\tilde{x}_{m, l, n}$ is the noisy data, $\hat{a}_{m, k}, \hat{s}_{l, k}$, and $\hat{h}_{n, k}$ are the estimates of the $a_{m, k}, s_{l, k}$, and $h_{n, k}$, respectively.

With respect to Equations 18, 20, and 21, the matrices $\mathbf{S}_{0}, \overline{\mathbf{A}}$, and $\mathbf{H}$ are conditionally updated with least squares, respectively. TALS is quite easy to implement and guaranteed to converge. In this paper, we use the complex parallel factor analysis (COMFAC) algorithm [25] for trilinear decomposition. COMFAC algorithm is essentially a fast implementation of TALS.

\subsection{Identifiability of trilinear decomposition}

In this subsection, we discuss the identifiability of trilinear decomposition.

Theorem 1. [24]: We consider $\mathbf{V}_{n}=\overline{\mathbf{A}} D_{n}(\mathbf{H}) \mathbf{S}_{0}+\mathbf{N}_{n}$, where $\overline{\mathbf{A}} \in i^{2 M \times K}, \mathbf{H} \in i^{4 \times K}$, and $\mathbf{S}_{0} \in i^{L \times K}$. If $k_{\mathbf{H}}+k_{\mathbf{S}_{0}}+k_{A} \geq$ $2 K+2\left(k_{\mathbf{H}}\right.$ is $k$-rank of the matrix $\mathbf{H}$ [24]), then $\overline{\mathbf{A}}, \mathbf{S}_{0}$, and $\mathbf{H}$ are unique up to the permutation and scaling of columns, which indicates any other triple $\hat{\overline{\mathbf{A}}}, \hat{\mathbf{S}}_{0}, \hat{\mathbf{H}}$ that construct $\mathbf{V}_{n}(n=1, \ldots, 4)$ is related to $\overline{\mathbf{A}}, \mathbf{S}_{0}$, and $\mathbf{H}$ via

$$
\begin{aligned}
\hat{\overline{\mathbf{A}}} & =\overline{\mathbf{A}} \boldsymbol{\Pi} \boldsymbol{\Delta}_{\mathbf{1}}+\mathbf{N}_{1}, \hat{\mathbf{S}}_{0}=\mathbf{S}_{0} \boldsymbol{\Pi} \boldsymbol{\Delta}_{\mathbf{2}}+\mathbf{N}_{2}, \hat{\mathbf{H}} \\
& =\mathbf{H} \boldsymbol{\Pi} \boldsymbol{\Delta}_{\mathbf{3}}+\mathbf{N}_{3} .
\end{aligned}
$$

where $\Pi$ is a permutation matrix, and $\Delta_{1}, \Delta_{2}$, and $\Delta_{3}$ are diagonal scaling matrices which satisfy $\Delta_{1}, \Delta_{2}, \Delta_{3}=\mathbf{I}_{K} . \mathbf{N}_{1}$, $\mathbf{N}_{2}$, and $\mathbf{N}_{3}$ are estimation errors.

Generically, the matrices are full $k$-rank, and then the identifiable condition becomes

$$
\min (4, K)+\min (L, K)+\min (2 M, K) \geq 2 K+2 .
$$

\subsection{Two-dimensional DOA estimation algorithm}

For the received noisy signal, we attain $\hat{\mathbf{H}}=\mathbf{H} \boldsymbol{\Pi} \boldsymbol{\Delta}_{\mathbf{3}}+\mathbf{N}_{3}$ through PARAFAC decomposition. The matrix $\hat{\mathbf{H}}$ is processed through normalization, which also resolves the scale ambiguity, to get the matrix $\widehat{\mathbf{H}}$. The $i$ th column of $\widehat{\mathbf{H}}$ is $\widehat{\mathbf{h}}_{i}$. In the case of noise absence, $\widehat{\mathbf{h}}_{i}$ should be

$$
\widehat{\mathbf{h}}_{i}=\left[\begin{array}{c}
1 \\
\cos \phi_{i} \cos \varphi_{i} \\
\sin \phi_{i} \cos \varphi_{i} \\
\sin \varphi_{i}
\end{array}\right]
$$

Finally, the elevation and azimuth angles are estimated by

$$
\begin{aligned}
& \hat{\varphi}_{i}=\sin ^{-1}\left(\widehat{\mathbf{h}}_{i}(4)\right) . \\
& \hat{\phi}_{i}=\operatorname{angle}\left(\widehat{\mathbf{h}}_{i}(2)+j \widehat{\mathbf{h}}_{i}(3)\right) .
\end{aligned}
$$

A NC-PARAFAC-based 2D-DOA estimation for acoustic vector-sensor array is proposed in this paper. The detailed steps are shown as follows:

Step 1. Construct the data matrix $\mathbf{V}$ via Equations 8 and 9 .

Step 2. Initial the matrices $\overline{\mathbf{A}}, \mathbf{S}_{0}$, and $H$.

Step 3. LS update for the matrix $\mathbf{S}_{0}$ according to Equation 18.

Step 4. LS update for the matrix $\overline{\mathbf{A}}$ according to Equation 20.

Step 5. LS update for the matrix $\mathbf{H}$ according to Equation 21.

Step 6. Repeat Step 3 to Step 5 until convergence.

Step 7. Estimate 2D-DOA according to the estimated matrix $\hat{\mathbf{H}}$.

Remark 2 Since $\hat{\varphi}_{i}$ and $\hat{\phi}_{i}$ (the estimation of elevation angle and azimuth angle) are estimated via $\widehat{\mathbf{h}}_{i}$ (the $i$ th column of $\widehat{\mathbf{H}})$, the 2D angle estimation can be paired automatically.

Remark 3. Random initialization is used for the proposed algorithm, and we also employ two slices and ESPRIT algorithm to obtain the initial estimation of parameter matrices.

Remark 4. The number of sources is pre-known, and it can be estimated by some methods in [26-29].

\subsection{Complexity analysis and advantages of the proposed algorithm}

The algorithm that we propose can have a lower computational complexity compared to PARAFAC algorithm. PARAFAC algorithm in [15] requires $O\left(n K^{3}+4 n M L K\right)$, where $n$ is the number of TALS iterations, while our NC-PARAFAC algorithm requires $O\left(n K^{3} / 4+2 n M L K\right)$. Our algorithm has a heavier computational load than 
ESPRIT algorithm, and the complexity of ESPRIT is $O\left(16 M^{2} L+64 M^{3}+K^{3}\right)$.

Figure 2 shows the complexity comparison among three algorithms with $n=40, K=3$, and $M=8$. From Figure 2 we find that our algorithm has lower complexity than PARAFAC and higher complexity than ESPRIT.

The proposed algorithm in this paper has the following advantages:

1. The angle estimation performance of the proposed algorithm is better than that of ESPRIT algorithm, PM, and PARAFAC algorithm.

2. The proposed algorithm can be suitable for arbitrary array subjected to unknown locations.

3. The proposed algorithm can achieve automatically paired two-dimensional angle estimation.

4. The proposed algorithm has lower computational complexity than PARAFAC algorithm.

\section{Crámer-Rao bound}

There are some differences between the CRB of noncircular signal DOA estimation and that of circular signal DOA estimation. The parameters which are needed to estimate can be expressed as

$$
\begin{array}{r}
\zeta=\left[\varphi_{1}, \ldots, \varphi_{K}, \phi_{1}, \ldots, \phi_{K}, \mathbf{s}_{R}^{T}(1), \ldots, \mathbf{s}_{R}^{T}(L),\right. \\
\left.\mathbf{s}_{I}^{T}(1), \ldots, \mathbf{s}_{I}^{T}(L), \sigma^{2}\right]^{T} .
\end{array}
$$

where $\mathbf{s}_{R}(t), t=1, \ldots, L$ and $\mathbf{s}_{I}(t), t=1, \ldots, L$ denote the real and imaginary parts of $s(t)$, respectively.

According to Equation 6, the output with $L$ snapshots is rewritten as

$$
\mathbf{y}=\left[\mathbf{x}_{E}^{T}(1), \ldots, \mathbf{x}_{E}^{T}(L), \mathbf{x}_{E}^{H}(1), \ldots, \mathbf{x}_{E}^{H}(L)\right] .
$$

where $\mathbf{x}_{\mathrm{E}}(l)$ is the $l$ th column of the matrix $\mathbf{X}_{E}$.

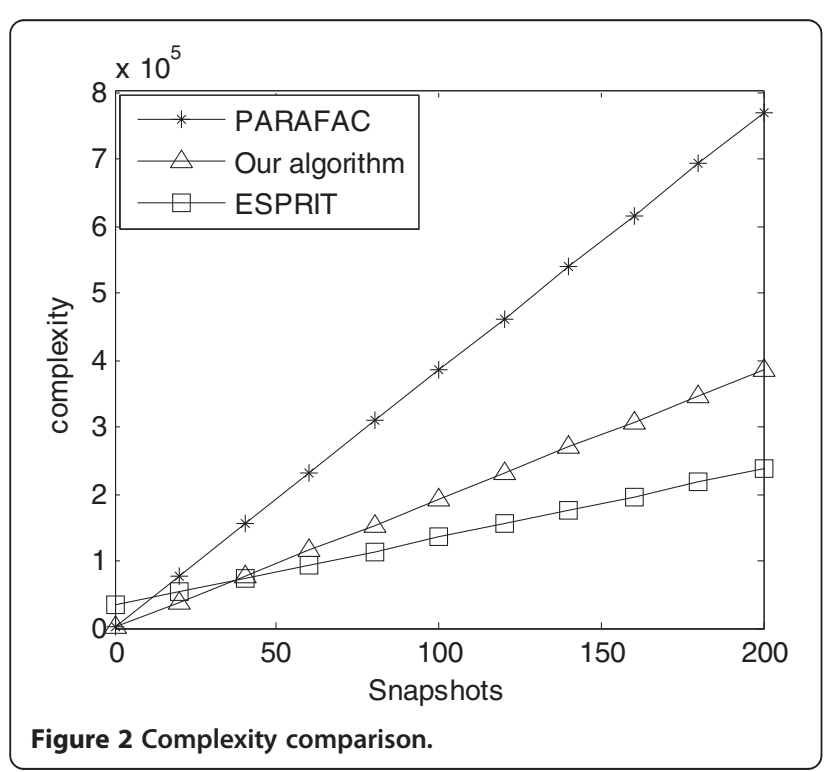

The mean $\boldsymbol{\mu}$ and the covariance matrix $\boldsymbol{\Gamma}$ of $\mathbf{y}$ are

$$
\boldsymbol{\mu}=\left[\begin{array}{c}
\mathbf{A}_{E} \mathbf{s}(1) \\
\vdots \\
\mathbf{A}_{E} \mathbf{s}(L) \\
\mathbf{A}_{E}^{*} \mathbf{s}^{*}(1) \\
\vdots \\
\mathbf{A}_{E}^{*} \mathbf{s}^{*}(L)
\end{array}\right], \cdots \boldsymbol{\Gamma}=\left[\begin{array}{ccc}
\sigma^{2} \mathbf{I} & & 0 \\
& \ddots & \\
0 & & \sigma^{2} \mathbf{I}
\end{array}\right]
$$

From [30], the $(i, j)$ element of the CRB matrix $\left(\mathbf{P}_{c r}\right)$ is expressed as

$$
\left[\mathbf{P}_{c r}^{-1}\right]_{i j}=\operatorname{tr}\left[\boldsymbol{\Gamma}^{-1} \boldsymbol{\Gamma}_{i}^{\prime} \boldsymbol{\Gamma}^{-1} \boldsymbol{\Gamma}_{j}^{\prime}\right]+2 \operatorname{Re}\left[\boldsymbol{\mu}_{i}^{\prime *} \boldsymbol{\Gamma}^{-1} \boldsymbol{\mu}_{j}^{\prime}\right]
$$

where $\boldsymbol{\Gamma}_{i}^{\prime}$ and $\boldsymbol{\mu}_{i}^{\prime}$ are the derivative of $\boldsymbol{\Gamma}$ and $\boldsymbol{\mu}$ on the $i$ th element of $\zeta$, respectively. The covariance matrix is just related to $\sigma^{2}$, so the first part of Equation 31 can be ignored. Then the $(i, j)$ element of the CRB matrix $\left(\mathbf{P}_{c r}\right)$ can be rewritten as

$$
\left[\mathbf{P}_{c r}^{-1}\right]_{i j}=2 \operatorname{Re}\left[\boldsymbol{\mu}_{i}^{\prime *} \boldsymbol{\Gamma}^{-1} \boldsymbol{\mu}_{j}^{\prime}\right]
$$

According to Equation 32,

$$
\frac{\partial \boldsymbol{\mu}}{\partial \varphi_{k}}=\left[\begin{array}{c}
\frac{\partial \mathbf{A}_{E}}{\partial \varphi_{k}} \mathbf{s}(1) \\
\vdots \\
\frac{\partial \mathbf{A}_{E}}{\partial \varphi_{k}} \mathbf{s}(L) \\
\frac{\partial \mathbf{A}_{E}{ }^{*}}{\partial \varphi_{k}} \mathbf{s}^{*}(1) \\
\vdots \\
\frac{\partial \mathbf{A}_{E}{ }^{*}}{\partial \varphi_{k}} \mathbf{s}^{*}(L)
\end{array}\right]=\left[\begin{array}{c}
\mathbf{d}_{k \varphi} \mathbf{s}_{k}(1) \\
\vdots \\
\mathbf{d}_{k \varphi} \mathbf{s}_{k}(L) \\
\mathbf{d}_{k \varphi} \mathbf{s}_{k}{ }^{*}(1) \\
\vdots \\
\mathbf{d}_{k \varphi}{ }^{*} \mathbf{s}_{k}{ }^{*}(L)
\end{array}\right], \quad k=1, \ldots, K
$$

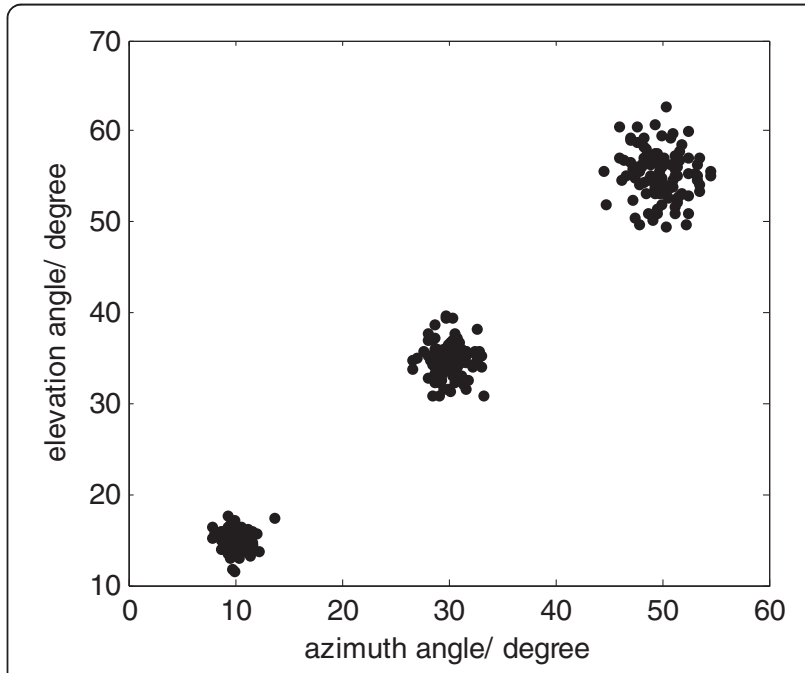

Figure 3 Angle estimation performance of the proposed algorithm with SNR $=0 \mathrm{~dB}$. 


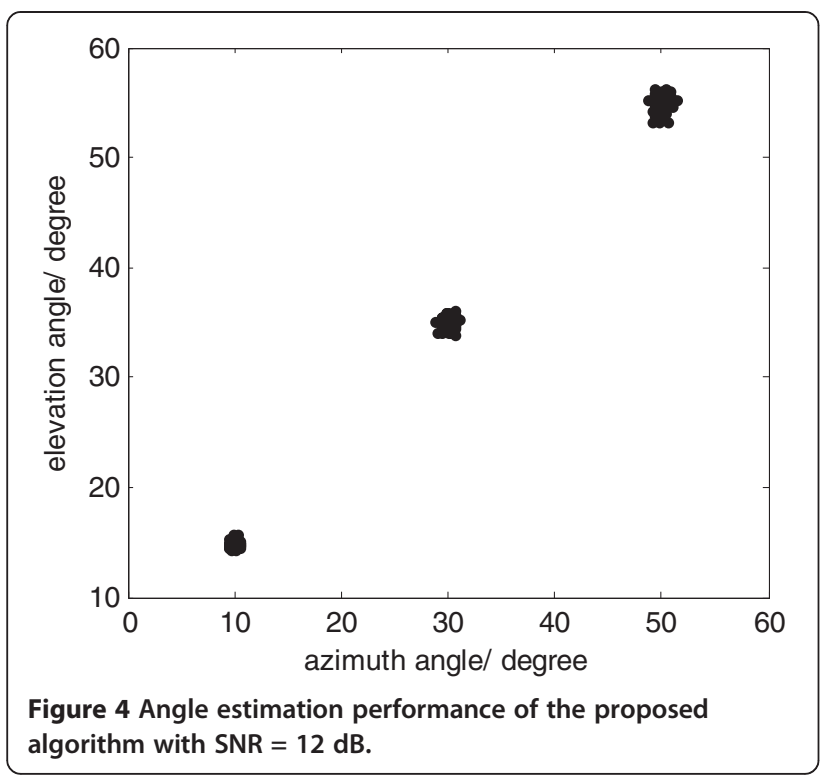

$$
\frac{\partial \boldsymbol{\mu}}{\partial \phi_{k}}=\left[\begin{array}{c}
\frac{\partial \mathbf{A}_{E}}{\partial \phi_{k}} \mathbf{s}(1) \\
\vdots \\
\frac{\partial \mathbf{A}_{E}}{\partial \phi_{k}} \mathbf{s}(L) \\
\frac{\partial \mathbf{A}_{E}^{*}}{\partial \phi_{k}} \mathbf{s}^{*}(1) \\
\vdots \\
\frac{\partial \mathbf{A}_{E}{ }^{*}}{\partial \phi_{k}} \mathbf{s}^{*}(L)
\end{array}\right]=\left[\begin{array}{c}
\mathbf{d}_{k \phi} \mathbf{s}_{k}(1) \\
\vdots \\
\mathbf{d}_{k \phi} \mathbf{s}_{k}(L) \\
\mathbf{d}_{k \phi}{ }^{*} \mathbf{s}_{k}{ }^{*}(1) \\
\vdots \\
\mathbf{d}_{k \phi}{ }^{*} \mathbf{s}_{k}{ }^{*}(L)
\end{array}\right], \quad k=1, \ldots, K .
$$

where $\mathbf{s}_{k}(t)$ is the $k$ th element of $\mathbf{s}(t)$, and

$$
\mathbf{d}_{k \varphi}=\frac{\partial \mathbf{a}_{E}\left(\varphi_{k}, \phi_{k}\right)}{\partial \varphi_{k}}
$$

$$
\mathbf{d}_{k \phi}=\frac{\partial \mathbf{a}_{E}\left(\varphi_{k}, \phi_{k}\right)}{\partial \phi_{k}}
$$

where $\mathbf{a}_{E}\left(\varphi_{k}, \varnothing_{k}\right)$ is the $k$ th column of $\mathbf{A}_{E}$.

Let

$$
\Delta \triangleq\left[\begin{array}{cccccc}
\mathbf{d}_{1 \varphi} \mathbf{s}_{1}(1) & \cdots & \mathbf{d}_{K \varphi} \mathbf{s}_{K}(1) & \mathbf{d}_{1 \phi} \mathbf{s}_{1}(1) & \cdots & \mathbf{d}_{K \phi} \mathbf{s}_{K}(1) \\
\vdots & & \vdots & \vdots & & \vdots \\
\mathbf{d}_{1 \varphi} \mathbf{s}_{1}(L) & \cdots & \mathbf{d}_{K \varphi} \mathbf{s}_{K}(L) & \mathbf{d}_{1 \phi} \mathbf{s}_{1}(L) & \cdots & \mathbf{d}_{K \phi} \mathbf{s}_{1}(L)
\end{array}\right]
$$

and

$$
\mathbf{G} \triangleq\left[\begin{array}{ccc}
\mathbf{A}_{E} & & 0 \\
& \ddots & \\
0 & & \mathbf{A}_{E}
\end{array}\right], \quad \mathbf{s}=\left[\begin{array}{c}
\mathbf{s}(1) \\
\vdots \\
\mathbf{s}(L)
\end{array}\right]
$$

then $\boldsymbol{\mu}=\left[\begin{array}{c}\mathbf{G s} \\ \mathbf{G}^{*} \mathbf{s}^{*}\end{array}\right]$, and

$$
\frac{\partial \boldsymbol{\mu}}{\partial \mathbf{s}_{R}{ }^{T}}=\left[\begin{array}{c}
\mathbf{G} \\
\mathbf{G}^{*}
\end{array}\right], \quad \frac{\partial \boldsymbol{\mu}}{\partial \mathbf{s}_{I}^{T}}=\left[\begin{array}{c}
j \mathbf{G} \\
-j \mathbf{G}^{*}
\end{array}\right]
$$

According to Equations 35, 36, and 37,

$$
\frac{\partial \boldsymbol{\mu}}{\partial \zeta^{T}}=\left[\begin{array}{cccc}
\Delta & \mathbf{G} & j \mathbf{G} & 0 \\
\Delta^{*} & \mathbf{G}^{*} & -j \mathbf{G}^{*} & 0
\end{array}\right]
$$

Equation 32 can be denoted by

$$
2 \operatorname{Re}\left\{\frac{\partial \boldsymbol{\mu}^{*}}{\partial \zeta} \Gamma^{-1} \frac{\partial \boldsymbol{\mu}}{\partial \boldsymbol{\zeta}^{T}}\right\}=\left[\begin{array}{ll}
\mathbf{J} & 0 \\
0 & 0
\end{array}\right]
$$

where

$$
\mathbf{J} \triangleq \frac{2}{\sigma^{2}} \operatorname{Re}\left\{\left[\begin{array}{c}
\Delta^{H}, \Delta^{T} \\
\mathbf{G}^{H}, \mathbf{G}^{T} \\
-j \mathbf{G}^{H}, j \mathbf{G}^{T}
\end{array}\right]\left[\begin{array}{ccc}
\Delta & \mathbf{G} & j \mathbf{G} \\
\Delta^{*} & \mathbf{G}^{*} & -j \mathbf{G}^{*}
\end{array}\right]\right\} .
$$
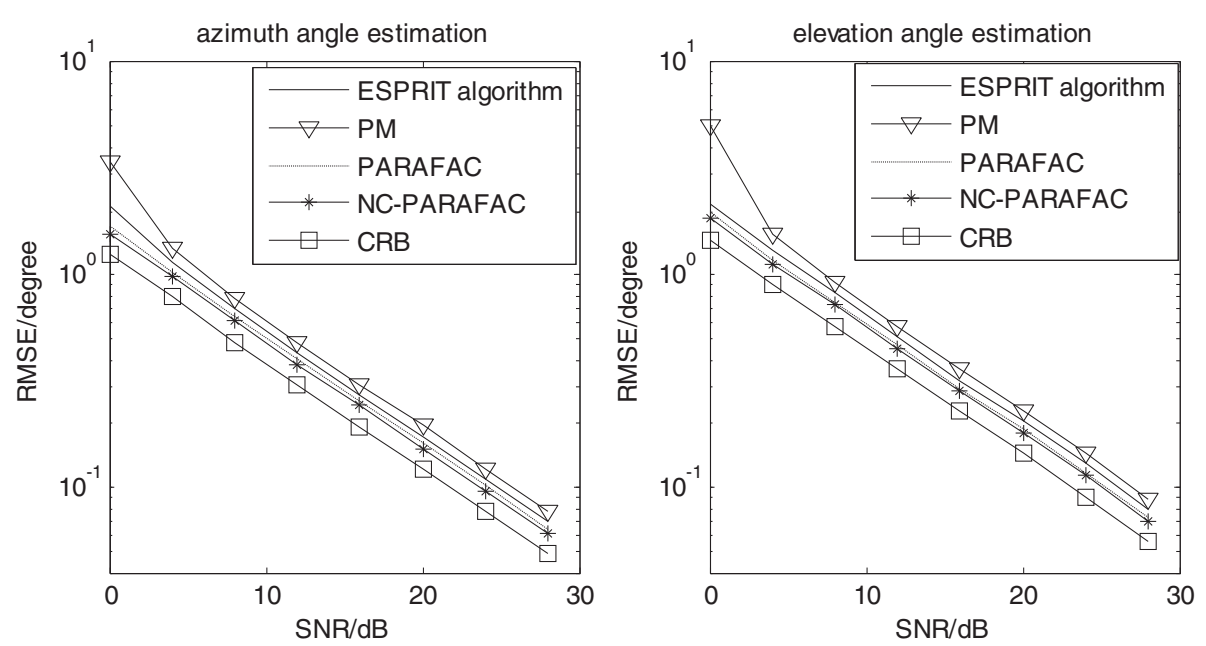

Figure 5 Angle estimation performance comparison $(M=8, L=200$, and $K=3)$. 

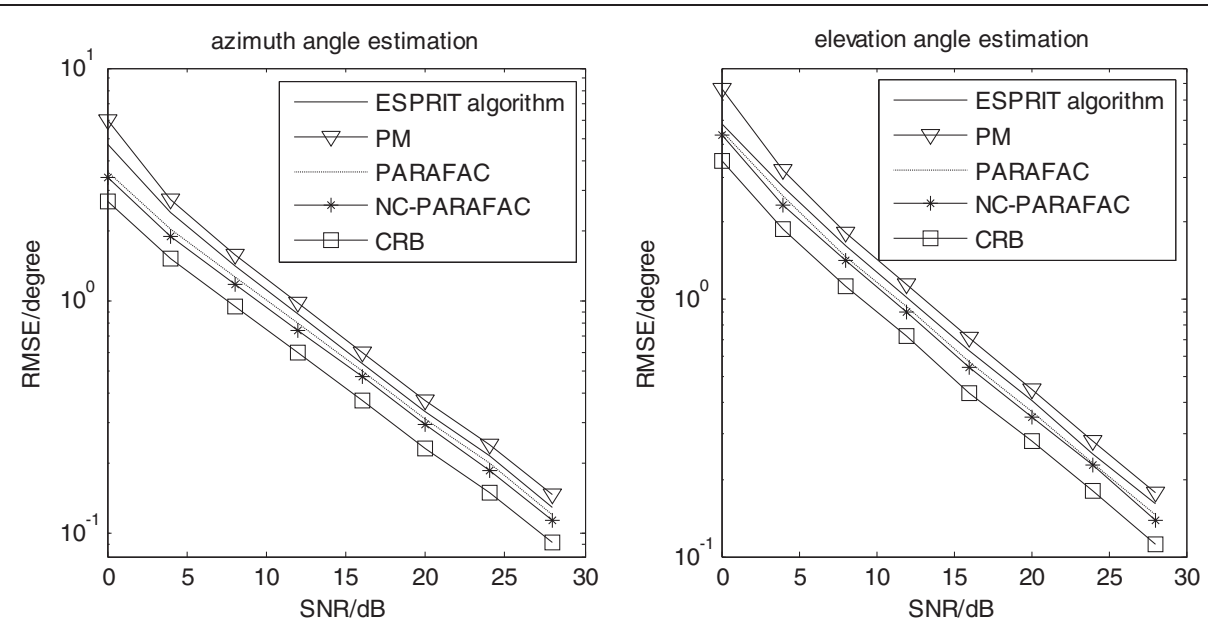

Figure 6 Angle estimation performance comparison $(M=6, L=100$, and $K=3)$.

Thus, we just consider the elements related to the angles in $\mathbf{J}^{-1}$. Define

$$
\begin{gathered}
\mathbf{B} \triangleq\left(\mathbf{G}^{H} \mathbf{G}\right)^{-1} \mathbf{G}^{H} \boldsymbol{\Delta} \\
\mathbf{F} \triangleq\left[\begin{array}{ccc}
\mathbf{I} & 0 & 0 \\
-\mathbf{B}_{R} & \mathbf{I} & 0 \\
-\mathbf{B}_{I} & 0 & \mathbf{I}
\end{array}\right]
\end{gathered}
$$

We demonstrate that

$$
\begin{aligned}
{\left[\begin{array}{rll}
\boldsymbol{\Delta} & \mathbf{G} & j \mathbf{G}
\end{array}\right] \mathbf{F} } & =\left[\begin{array}{lll}
(\boldsymbol{\Delta}-\mathbf{G B}) & \mathbf{G} & j \mathbf{G}
\end{array}\right] \\
& =\left[\begin{array}{lll}
\mathbf{\Pi}_{\mathbf{G}}^{\perp} \boldsymbol{\Delta} & \mathbf{G} & j \mathbf{G}
\end{array}\right]
\end{aligned}
$$

$$
\begin{aligned}
{\left[\begin{array}{rll}
\boldsymbol{\Delta}^{*} & \mathbf{G}^{*} & -j \mathbf{G}^{*}
\end{array}\right] \mathbf{F} } & =\left[\begin{array}{lll}
\left(\boldsymbol{\Delta}^{*}-\mathbf{G}^{*} \mathbf{B}^{*}\right) & \mathbf{G}^{*} & -j \mathbf{G}^{*}
\end{array}\right] \\
& =\left[\begin{array}{lll}
\boldsymbol{\Pi}_{\mathbf{G}^{*}}^{+} \boldsymbol{\Delta}^{*} & \mathbf{G}^{*} & -j \mathbf{G}^{*}
\end{array}\right]
\end{aligned}
$$

where $\boldsymbol{\Pi}_{\mathbf{G}}^{\perp}=\mathbf{I}-\mathbf{G}\left(\mathbf{G}^{H} \mathbf{G}\right)^{-1} \mathbf{G}^{H}$ and $\mathbf{G}^{H} \boldsymbol{\Pi}_{\mathbf{G}}^{\perp}=0$.

$$
\begin{aligned}
\mathbf{F}^{T} \mathbf{J F} & =\frac{2}{\sigma^{2}} \operatorname{Re}\left\{\mathbf{F}^{H}\left[\begin{array}{c}
\Delta^{H}, \boldsymbol{\Delta}^{T} \\
\mathbf{G}^{H}, \mathbf{G}^{T} \\
-j \mathbf{G}^{H}, j \mathbf{G}^{T}
\end{array}\right]\left[\begin{array}{ccc}
\Delta & \mathbf{G} & j \mathbf{G} \\
\boldsymbol{\Delta}^{*} & \mathbf{G}^{*} & -j \mathbf{G}^{*}
\end{array}\right] \mathbf{F}\right\} \\
& =\frac{2}{\sigma^{2}} \operatorname{Re}\left\{\left[\begin{array}{c}
\Delta^{H} \boldsymbol{\Pi}_{\mathbf{G}}^{\perp}, \boldsymbol{\Delta}^{T} \boldsymbol{\Pi}_{\mathbf{G}}^{\perp} \\
\mathbf{G}^{H}, \mathbf{G}^{T} \\
-j \mathbf{G}^{H}, j \mathbf{G}^{T}
\end{array}\right]\left[\begin{array}{lll}
\boldsymbol{\Pi}_{\mathbf{G}}^{\perp} \boldsymbol{\Delta} & \mathbf{G} & j \mathbf{G} \\
\boldsymbol{\Pi}_{\mathbf{G}^{*}}^{\perp} \boldsymbol{\Delta}^{*} & \mathbf{G}^{*} & -j \mathbf{G}^{*}
\end{array}\right]\right\}, \\
& =\frac{4}{\sigma^{2}} \operatorname{Re}\left\{\left[\begin{array}{ccc}
\Delta^{H} \boldsymbol{\Pi}_{\mathbf{G}}^{\perp} \boldsymbol{\Delta} & 0 & 0 \\
0 & \mathbf{G}^{H} \mathbf{G} & j \mathbf{G}^{H} \mathbf{G} \\
0 & -j \mathbf{G}^{H} \mathbf{G} & \mathbf{G}^{H} \mathbf{G}
\end{array}\right]\right\}
\end{aligned}
$$
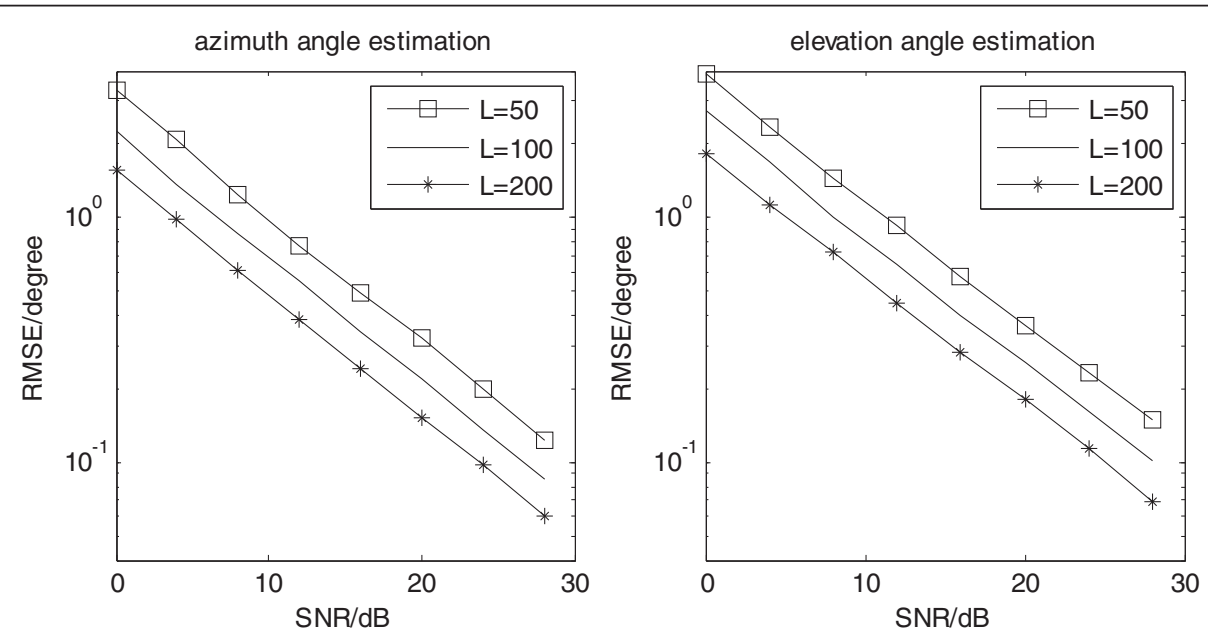

Figure 7 Angle estimation performance with different values of $L$. 

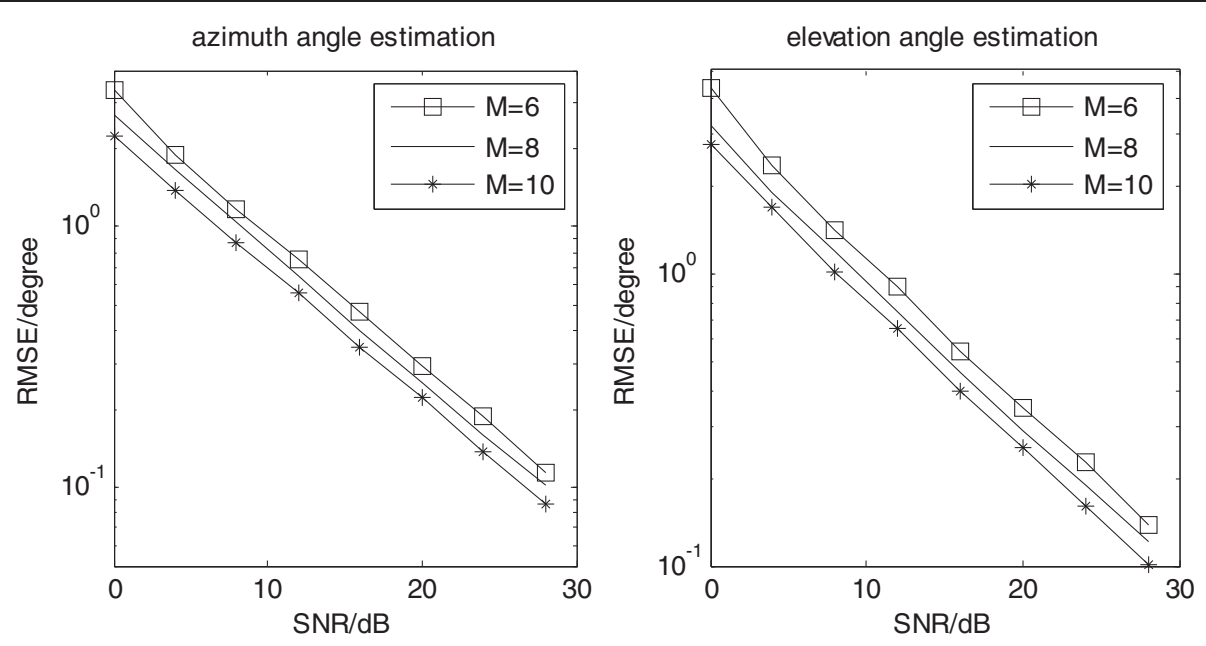

Figure 8 Angle estimation performance with different values of $M$.

so $\mathbf{J}^{-1}$ is written as

$$
\begin{aligned}
& \mathbf{J}^{-1}=\mathbf{F}\left(\mathbf{F}^{T} \mathbf{J F}\right)^{-1} \mathbf{F}^{T} \\
& =\frac{\sigma^{2}}{4}\left[\begin{array}{ccc}
\mathbf{I} & 0 & 0 \\
-\mathbf{B}_{R} & \mathbf{I} & 0 \\
-\mathbf{B}_{I} & 0 & \mathbf{I}
\end{array}\right]\left[\begin{array}{ccc}
\operatorname{Re}\left(\boldsymbol{\Delta}^{H} \boldsymbol{\Pi}_{\mathbf{G}}^{\perp} \boldsymbol{\Delta}\right) & 0 & 0 \\
0 & \kappa & \kappa \\
0 & \kappa & \kappa
\end{array}\right] \\
& {\left[\begin{array}{ccc}
\mathbf{I} & -\mathbf{B}_{R}^{T} & -\mathbf{B}_{I}^{T} \\
0 & \mathbf{I} & 0 \\
0 & 0 & \mathbf{I}
\end{array}\right]} \\
& =\left[\begin{array}{ccc}
\frac{\sigma^{2}}{4}\left[\operatorname{Re}\left(\Delta^{H} \boldsymbol{\Pi}_{\mathbf{G}}^{\perp} \boldsymbol{\Delta}\right)\right]^{-1} & \kappa & \kappa \\
\kappa & \kappa & \kappa \\
\kappa & \kappa & \kappa
\end{array}\right]
\end{aligned}
$$

where $\kappa$ denotes the part we do not concern. Until now, we give the $C R B$ matrix as follows

$$
\mathrm{CRB}=\frac{\sigma^{2}}{4}\left[\operatorname{Re}\left(\Delta^{H} \boldsymbol{\Pi}_{\mathbf{G}}^{\perp} \boldsymbol{\Delta}\right)\right]^{-1} .
$$

After further simplification, we rewrite the CRB matrix

$$
\mathrm{CRB}=\frac{\sigma^{2}}{4 L}\left\{\operatorname{Re}\left[\left(\mathbf{D}^{H} \Pi_{\mathbf{A}_{E}}^{\perp} \mathbf{D}\right) \odot \mathbf{P}^{T}\right]\right\}^{-1}
$$

where $\odot$ stands for Hadamard product. $\quad \mathbf{P}=\frac{1}{L}$ $\sum_{l=1}^{L} \mathbf{s}(l) \mathbf{s}^{H}(l), \quad \mathbf{D}=\left[\mathbf{e}_{1}, \mathbf{e}_{2}, \cdots, \mathbf{e}_{K}, \mathbf{f}_{1}, \mathbf{f}_{2}, \cdots, \mathbf{f}_{K}\right], \mathbf{e}_{k}=\partial(\mathbf{a}$ $\left.\left(\varphi_{k}\right) \otimes \mathbf{h}_{k}\right) / \partial \phi_{k}, \mathbf{f}_{k}=\partial\left(\mathbf{a}\left(\varphi_{k}\right) \otimes \mathbf{h}_{k}\right) / \partial \varphi_{k}$, and $\mathbf{h}_{k}=\left[1, \mathbf{u}_{k}^{T}\right]^{T}$. $\Pi_{\mathbf{A}_{E}}^{\perp}=\mathbf{I}_{4 M}-\mathbf{A}_{E}\left(\mathbf{A}_{E}{ }^{H} \mathbf{A}_{E}\right)^{-1} \mathbf{A}_{E}{ }^{H} \sigma^{2}$ is the power of the noise.

\section{Simulation results}

We define root mean square error (RMSE) as

$$
\mathrm{RMSE}=1 / K \sum_{k=1}^{K} \sqrt{1 / N \sum_{n=1}^{N}\left(\hat{a}_{k, n}-a_{k}\right)^{2}}
$$

where $\hat{a}_{k, n}$ is the estimation of elevation angle/azimuth angle $a_{k}$ of the $n$th Monte Carlo trial. We assume that there are $K=3$ sources with angles $\left(\phi_{1}, \varphi_{1}\right)=\left(10^{\circ}, 15^{\circ}\right)$, $\left(\phi_{2}, \varphi_{2}\right)=\left(30^{\circ}, 35^{\circ}\right)$, and $\left(\phi_{3}, \varphi_{3}\right)=\left(50^{\circ}, 55^{\circ}\right)$.

Figures 3 and 4 depict angle estimation results of the proposed algorithm for all three sources with $M=10, L=$ 200, signal-to-noise ratio $(\mathrm{SNR})=0 \mathrm{~dB}$ and $M=10, L=$ 200 , SNR $=12 \mathrm{~dB}$, respectively. It is shown that the elevation and azimuth angles can be clearly observed.

Figures 5 and 6 present the comparisons among our NC-PARAFAC algorithm, ESPRIT algorithm, PARAFAC algorithm, and CRB. From Figures 5 and 6, we can find that the proposed algorithm has better angle estimation performance than ESPRIT algorithm and PM. Furthermore, our NC-PARAFAC algorithm has better angle

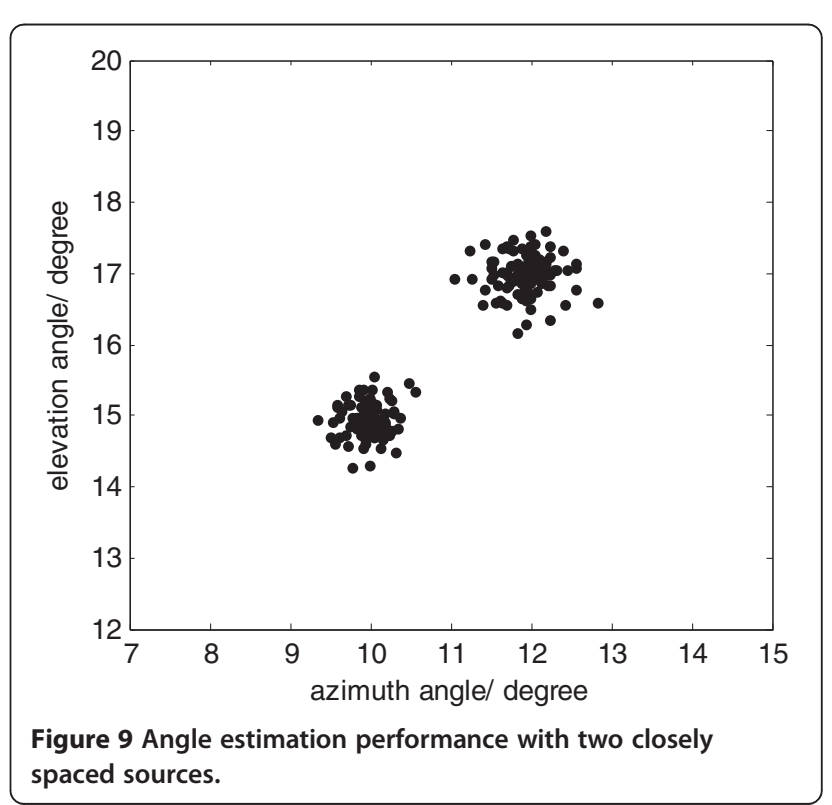


estimation performance than PARAFAC algorithm since it uses noncircularity to expand the array aperture. Our algorithm requires the noncircularity of signal, while ESPRIT algorithm and PARAFAC algorithm need no knowledge on the noncircularity.

Figure 7 presents angle estimation performance of the proposed algorithm with $M=10, K=3$ and different values of $L$. It is indicated in Figure 7 that the angle estimation performance of the proposed algorithm is improved with $L$ increasing.

Figure 8 shows angle estimation performance of the proposed algorithm with $L=100, K=3$ and different values of $M$. From Figure 8 , the angle estimation performance of the proposed algorithm is improved with the number of antennas increasing.

Figure 9 displays the angle estimation of the proposed algorithm with two closely spaced sources. We assume two close sources located at angles $\left(\phi_{1}, \varphi_{1}\right)=\left(10^{\circ}, 15^{\circ}\right)$, $\left(\phi_{2}, \varphi_{2}\right)=\left(12^{\circ}, 17^{\circ}\right) . M=12, J=100, K=2$, and SNR $=$ $12 \mathrm{~dB}$ are used in Figure 9. It implies that our algorithm works well for two closely spaced sources.

\section{Conclusions}

In this paper, we have presented an NC-PARAFAC algorithm for 2D-DOA estimation of noncircular signals for acoustic vector-sensor array. The proposed algorithm has better angle estimation performance than the ESPRIT algorithm, PARAFAC algorithm, and PM. Furthermore, the proposed algorithm has a lower computational complexity than PARAFAC algorithm. The proposed algorithm enhances the angle estimation performance via utilizing the noncircularity of the signals, and it can be suitable for arbitrary array subjected to unknown locations and achieve automatically paired two-dimensional angle estimation. We also analyze the complexity and derive the CRB of noncircular signal DOA estimation.

\section{Competing interests}

The authors declare that they have no competing interests.

\section{Acknowledgements}

This work is supported by China NSF grants $(61201208,61271327,61071164)$, Jiangsu Planned Projects for Postdoctoral Research Funds (1201039C), China Postdoctoral Science Foundation (2012M521099), Open project of the Key Laboratory of Underwater Acoustic Communication and Marine Information Technology (Xiamen University), Hubei Key Laboratory of Intelligent Wireless Communications (IWC2012002), Open project of the Key Laboratory of Nondestructive Testing (Nanchang Hangkong University), Open project of the Key Laboratory of Modern Acoustic of the Ministry of Education (Nanjing University), the Aeronautical Science Foundation of China (20120152001), by the Qing Lan Project, and the Fundamental Research Funds for the Central Universities (NS2013024, NZ2012010, kfjj120115, kfjj20110215).

\section{Author details}

${ }^{1}$ College of Electronic and Information Engineering, Nanjing University of Aeronautics and Astronautics, Nanjing 210016, China. ${ }^{2}$ Nanjing Panda Electronics Group, Nanjing 210002, China. ${ }^{3}$ Laboratory of Modern Acoustic of Ministry of Education, Nanjing University, Nanjing, China.
Received: 8 November 2012 Accepted: 6 May 2013

Published: 20 May 2013

\section{References}

1. G Sun, Q Li, B Zhang, Acoustic vector sensor signal processing. Chinese J. Acoustics 25(1), 1-15 (2006)

2. A Nehorai, E Paldi, Acoustic vector-sensor array processing. IEEE Trans. Signal Process. 42(9), 2481-2491 (1994)

3. G Sun, D Yang, L Zhang, Maximum likelihood ratio detection and maximum likelihood DOA estimation based on the vector hydrophone. Acta Acustica 28(1), 66-72 (2003)

4. M Hawkes, A Nehorai, Acoustic vector-sensor beamforming and Capon direction estimation. IEEE Trans. Signal Process. 46(9), 2291-2304 (1998)

5. J He, Z Liu, Computationally efficient underwater acoustic 2-D source localization with arbitrarily spaced vector hydrophones at unknown locations using the propagator method. Multidim Syst Sign Process. 20, 285-296 (2009)

6. B Hochwald, A Nehorai, Identifiability in array processing models with vector-sensor applications. IEEE Trans. Signal Process. 44(1), 83-95 (1996)

7. KT Wong, MD Zoltowski, Closed-form underwater acoustic direction-finding with arbitrarily spaced vector hydrophones at unknown locations. IEEE Journal Oceanic Eng. 22(3), 566-575 (1997)

8. KT Wong, MD Zoltowski, Extended-Aperture underwater acoustic multisource azimuth/elevation direction-finding using uniformly but sparsely spaced vector hydrophones. IEEE J. Oceanic Eng. 22(4), 659-672 (1997)

9. J He, S Jiang, J Wang, Z Liu, Direction finding in spatially correlated noise fields with arbitrarily-spaced and far-separated subarrays at unknown locations. IET Radar Sonar \& Navigat. 3(3), 278-284 (2009)

10. KT Wong, MD Zoltowski, Root-MUSIC-based azimuth-elevation angle-of -arrival estimation with uniformly spaced but arbitrarily oriented velocity hydrophones. IEEE Trans. Signal Process. 47(12), 3250-3260 (1999)

11. KT Wong, MD Zoltowski, Self-initiating MUSIC-based direction finding in underwater acoustic particle velocity-field beamspace. IEEE Journal Oceanic Eng. 25(2), 262-273 (2000)

12. Y Wang, J Zhang, B Hu, J He, Hypercomplex model of acoustic vector sensor array with its application for the high resolution two dimensional direction of arrival estimation, in Proc. IEEE Instrumentation \& Measurement Technol. Conf. (IMTC' 2008) (, Victoria). 12-15 May 2008, pp. 1-5

13. Z Liu, X Ruan, J He, Efficient 2-D DOA estimation for coherent sources with a sparse acoustic vector-sensor array. Multidim. Syst. Signal Process. 24, 105-120 (2013)

14. X Zhang, M Zhou, H Chen, J Li, Two-dimensional DOA estimation for acoustic vector-sensor array using a successive MUSIC. Multidim Syst Sign Process (2013). doi:10.1007/s11045-012-0219-y. http://link.springer.com/ content/pdf/10.1007\%2Fs11045-012-0219-y.pdf.

15. X Zhang, J Li, H Chen, D Xu, Trilinear decomposition-based twodimensional DOA estimation algorithm for arbitrarily spaced acoustic vector-sensor array subjected to unknown locations. Wireless Pers Commun. 67(4), 859-877 (2012)

16. Y Yuan, B Zhang, D Fan, G Tong, DFT and PSD for estimating DOA with an active acoustic array. IEEE Int. Conf. Automat. \& Logistics (ICAL' 2008), 694-699 (2008)

17. KP Arunkumar, GV Anand, Multiple source localization in shallow ocean using a uniform linear horizontal array of acoustic vector sensors, in 2007 IEEE Intelligent Information Communication Technologies for Better Human Life (TENCON 2007) (, Taipei). 30 Oct.-2 Nov. 2007, pp. 1-4

18. PK Tam, KT Wong, Cramer-Rao bounds for direction finding by an acoustic vector sensor under nonideal gain-phase responses. IEEE Sensors Journa. 9(8), 969-982 (2009)

19. A Abdi, H Guo, Signal correlation modeling in acoustic vector sensor arrays. IEEE Trans. Signal Process. 57(3), 892-903 (2009)

20. M Hawkes, A Nehorai, Wideband source localization using a distributed acoustic vector-sensor array. IEEE Trans. Signal Process. 51(6), 1479-1491 (2003)

21. Z Nan, CC Swee, BAL Chew, Vector hydrophone array development and its associated DOA estimation algorithms. Asia Pacific OCEANS 2006, 1-5 (2006)

22. M Haardt, F Romer, Enhancements of unitary ESPRIT for noncircular sources, in Proceedings of IEEE International Conference on Acoustics, Speech, and Signal Processing, ed. by (, Montreal). 17-21 May 2004, pp. 101-104

23. $P$ Charge, $Y$ Wang, J Saillard, A non-circular sources direction finding method using polynomial rooting. Signal Process. 81(8), 1765-1770 (2001) 
24. ND Sidiropoulos, R Bro, GB Giannakis, Parallel factor analysis in sensor array processing. IEEE Trans. Signal Process. 48(8), 2377-2388 (2000)

25. R Bro, R Bro, ND Sidiropoulos, GB Giannakis, A fast least squares algorithm for separating trilinear mixtures, in Proceedings of ICA99 International Workshop on Independent Component Analysis and Blind Signal Separation (Aussois) 11-15 January 1999, pp. 289-294

26. A Di, Multiple sources location-a matrix decomposition approach. IEEE Trans on ASSP 35(4), 1086-1091 (1985)

27. M Wax, Detection of the number of coherent signals by the MDL principle. IEEE Trans On ASSP 37(8), 1190-1196 (1989)

28. T-J Shan, On smoothed rank profile tests in eigenstructure methods for directions-of-arrival estimation. IEEE Trans On ASSP 35(10), 1377-1385 (1987)

29. H-T Wu, Source number estimator using Gerschgorin disks. Acoustics, Speech, and Signal Process 4, 261-264 (1994)

30. P Stoica, A Nehorai, Performance study of conditional and unconditional direction-of-arrival estimation. IEEE Trans. Signal Process. 38(10), 1783-1795 (1990)

doi:10.1186/1687-6180-2013-107

Cite this article as: Zhang et al:: Noncircular-PARAFAC for 2D-DOA estimation of noncircular signals in arbitrarily spaced acoustic vectorsensor array subjected to unknown locations. EURASIP Journal on Advances in Signal Processing 2013 2013:107.

Submit your manuscript to a SpringerOpen ${ }^{\circ}$ journal and benefit from:

- Convenient online submission

- Rigorous peer review

- Immediate publication on acceptance

- Open access: articles freely available online

- High visibility within the field

- Retaining the copyright to your article 